\title{
Nucleotide sequence of the Streptococcus mutans gtfD gene encoding the glucosyltransferase-S enzyme
}

\author{
ORahito Honda, $\uparrow$ ChIaki Kato $\ddagger$ and Howard K. Kuramitsu $\ddagger^{*}$ \\ Department of Microbiology-Immunology, Northwestern University Medical-Dental Schools, Chicago, \\ Illinois 60611, USA
}

(Received 7 March 1990; revised 13 June 1990; accepted 27 June 1990)

\begin{abstract}
The nucleotide sequence of the Streptococcus mutans GS-5 gtfD gene coding for the glucosyltransferase which synthesizes water-soluble glucan (GTF-S) has been determined. The complete gene contains 4293 base pairs and the unprocessed protein is composed of 1430 amino acids with a molecular mass of $159814 \mathrm{Da}$. The amino terminus of the unprocessed protein resembles the signal sequences of other extracellular proteins secreted by $S$. mutans and that of the GTF-I secreted by Streptococcus downei. In addition, the GTF-S protein exhibits high amino acid similarity with the strain GS-5 enzymes responsible for insoluble glucan synthesis (GTF-I, GTF-SI) previously isolated and sequenced in this laboratory. These results indicate that all three gtf genes evolved from a common ancestral gene.
\end{abstract}

\section{Introduction}

Human dental caries is one of the most prevalent bacterial infections occurring in man (Gibbons \& van Houte, 1975). Although several different oral bacteria may contribute to caries formation, a variety of approaches have strongly implicated Streptococcus mutans as the principal etiological agent in this disease (Loesche, 1986). One of the important pathogenic properties of these organisms appears to be their ability to colonize tooth surfaces abundantly in the presence of dietary sucrose. This activity is dependent upon the production of extracellular glucosyltransferases (GTF; EC 2.4.1.5) which synthesize water-insoluble adherent glucans from sucrose. An examination of different strains of mutans streptococci has revealed two different classes of GTF: GTF-I, which synthesizes primarily water-insoluble glucans and GTF-S, which synthesizes predominantly water-soluble glucans (Kuramitsu \& Wondrack, 1983; Shimamura et al., 1983).

The genes which express the different GTF activities

\footnotetext{
† Present address: Hokkaido University School of Dentistry, Sapporo 060, Japan.

¥ Present address: Department of Pediatric Dentistry, University of Texas Health Science Center, San Antonio, Texas 78284, USA.

The nucleotide sequence data reported in this paper have been submitted to GenBank, and have been assigned the accession number M29296.
}

have recently been isolated (Gilpin et al., 1985; Aoki et al., 1986; Hanada \& Kuramitsu, 1988; Hanada \& Kuramitsu, 1989). Our laboratory has demonstrated that for one strain of S. mutans, GS-5, three distinct gtf genes are present on the chromosome (Aoki et al., 1986; Hanada \& Kuramitsu, 1988, 1989). Two of these, the tandemly-arranged $g t f B$ and $g t f C$ genes, code for the GTF-I and GTF-SI activities, respectively, which synthesize predominantly water-insoluble glucans. In addition, the $g t f D$ gene expresses the GTF-S activity of strain GS-5.

Recent nucleotide sequencing of the $g t f B$ and $g t f C$ genes (Shiroza et al., 1987; Ueda et al., 1988) has revealed extensive homology between the two gene products. In addition, both enzymes are homologous to the GTF-I protein from another species of mutans streptococci, Streptococcus downei (previously named Streptococcus sobrinus) strain MFe28 (Russell et al., 1988). It was, therefore, of interest to sequence the gtfD gene in order to initiate an examination of the molecular basis for the diverse synthetic activities of the GTF. The present communication describes the nucleotide sequence of the strain GS-5 gtfD gene and an analysis of the deduced amino acid sequence relative to the structures of the previously analysed GTF.

\section{Methods}

DNA manipulations. Plasmid pNH5 containing the intact $g t f D$ gene has been recently described (Hanada \& Kuramitsu, 1989). DNA 
isolation, endonuclease restriction digestion, ligation, and transformation of Escherichia coli were carried out as previously described (Aoki $e t$ al., 1986).

Nucleotide sequencing. Overlapping DNA fragments encompassing the entire $g t f D$ gene (Fig. 1) were isolated from pNH5 in either M13mp18 or M13mp19 (Bethesda Research Laboratories) with the exception of fragment 10B, which was isolated in pBlueScript SK (Strategene). A series of deleted bacteriophage clones was then prepared as described by Henikoff (1984). Single-stranded DNA fragments were isolated and sequenced using the dideoxy chaintermination procedure (Sangar et al., 1977) as previously described (Shiroza et al., 1987). Both strands of the $5.74 \mathrm{~kb}$ XbaI-KpnI DNA fragment containing the intact $g t f D$ gene were completely sequenced.

Sequence analysis. Nucleic acid and protein analyses were carried out using the Pustell sequence analysis programs (International Biotechnologies).

\section{Results}

\section{Nucleotide sequence of the gtfD gene}

The sequencing strategy depicted (Fig. 1) was used to determine the complete nucleotide sequence of the $5.74 \mathrm{~kb} S$. mutans GS-5 DNA fragment containing the gtfD gene. Only one of the two DNA strands contained a long open reading frame (ORF) capable of coding for the $155 \mathrm{kDa}$ GTF-S enzyme (Hanada \& Kuramitsu, 1989). The sequence detected contained 4293 bp and codes for a protein containing 1430 amino acids with an unprocessed molecular mass of $159814 \mathrm{Da}$ (Fig. 2).

Seven base pairs preceding the putative initiation codon ATG (position 435; Fig. 2) was the sequence GGAGG (nucleotides 423-427), which is homologous to the ribosome-binding site (Shine \& Dalgarno, 1974). In addition, the sequence TATAAT (positions 403-408), which is identical to the $E$. coli -10 promoter consensus sequence (Hawley \& McClure, 1983), was identified. However, no sequence homologous to the -35 consensus sequence could be identified in this region. It will be necessary to analyse mRNA transcripts from this region to define the precise transcription start site. As with many Gram-positive bacterial genes previously sequenced (Hollingshead et al., 1986; Shiroza \& Kuramitsu, 1988; Shiroza et al., 1987), the region upstream from the ribosome-binding site of the $g t f D$ gene $(180 \mathrm{bp})$ is AT-rich (73\% AT compared to $63 \%$ for the entire $S$. mutans chromosome; Hamada \& Slade, 1980). In addition, an inverted repeat sequence characteristic of rho-independent termination sequences was found $200 \mathrm{bp}$ downstream from the $g t f D$ stop codon (results not shown). In addition, an analysis of codon usage for the gtf $D$ gene revealed generally similar utilisation to that of the previously sequenced $g t f B$ and $g t f C$ genes (Shiroza $e t$ al., 1987; Ueda et al., 1988).

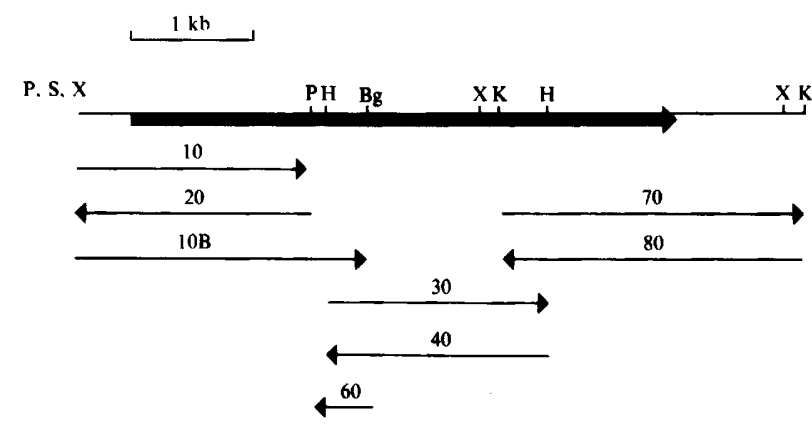

Fig. 1. Strategy for sequencing the $g t f D$ gene. The top line depicts the $S$. mutans strain GS-5 $5.74 \mathrm{~kb}$ insert from plasmid pNH5 containing the $g t f D$ gene. The thick line indicates the position of the gene, and the arrowhead indicates the direction of gene transcription. The overlapping fragments encompassing the gene, used for sequencing, are indicated below the insert with the directions of sequencing depicted. Bg, BglII ; H, HindIII; K, KpnI ; P, PstI; S, SalI; X, XbaI.

\section{Amino acid sequence of the GTF-S enzyme}

The deduced amino acid sequence of the $g t f D$ gene product indicated that the GTF-S protein contained an amino-terminal sequence typical of Gram-positive extracellular proteins (Hollingshead et al., 1986; von Heijne \& Abrahmson, 1989). This signal sequence region consisted of a strongly basic fourteen-amino-acid region followed by a relatively hydrophobic sequence (amino acids 15-29). The cleavage site for the signal peptidase has not yet been determined for the GTF-S protein. However, cleavage of the $159.8 \mathrm{kDa}$ unprocessed protein between amino acids $30-40$ would result in a protein with a molecular mass $(155 \mathrm{kDa})$ approximating that of the enzyme recently purified from $E$. coli clones containing the gtfD gene (Hanada \& Kuramitsu, 1989).

Like the GTF-I and GTF-SI proteins (Shiroza et al., 1987; Ueda et al., 1988), the GTF-S protein is highly hydrophilic (data not shown) with an estimated pI of 6.29. Furthermore, as with the GTF-I and GTF-SI enzymes of strain GS-5, no cysteine residues were identified in GTF-S.

\section{Sequence similarities of the GTF enzymes from S. mutans}

Recent results from this laboratory (Ueda et al., 1988) have indicated that the GTF-I and GTF-SI enzymes of $S$. mutans strain GS-5 share a high degree of identity for both the nucleotide and amino acid sequences. It was therefore of interest to compare the sequence of the GTF-S protein with these two proteins. An amino acid comparison (Fig. 3) revealed very strong similarity between the GTF-S and GTF-I proteins. This was especially true in the central regions of both proteins. Interestingly, the products of the $g t f B$ and $g t f C$ genes 


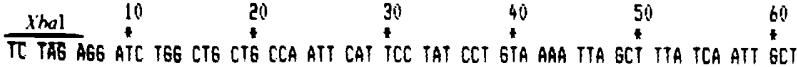
GIT ATT ICA CCT GCT GCA CTA AAC ACA GCT CTI CTC AGT CAG GTT TAI GGC GGA GAA TAT GAA TAT GGT TCE CGT CTE GIC ITA TIE ACA ACT GIT IIG TCT CTC CTE ACC ATT CCT ThaIII $190 \quad 200 \quad 250 \quad 220 \quad 230 \quad 240$ AAT AIG ACT GIT GCT AGC ITA ITG TAT ITA TAA AIT GIG ITA AAA GAT GTA ATT TAT AGC AhalII $260 \quad 270 \quad 280 \quad 290 \quad 300$

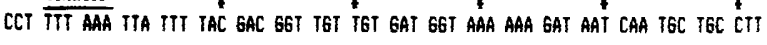

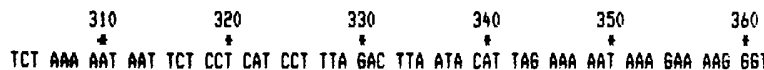
$370 \quad 380 \quad 390 \quad 400 \quad 410 \quad 420$

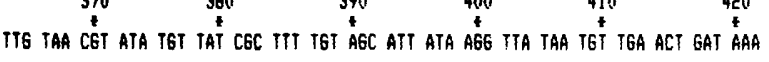

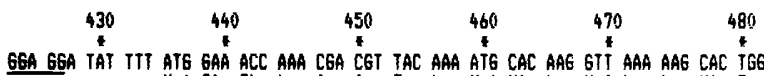

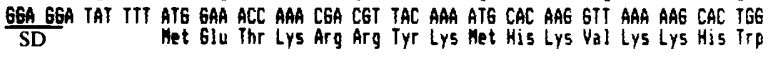

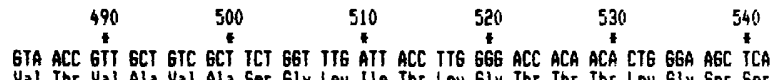
Val Thr val ala val Ala Ser Gly leu lle thr Leu Gly thr Thr thr Leu gly Ser Ser

$\begin{array}{llllll}550 & 560 & 570 & 580 & 590 & 600\end{array}$

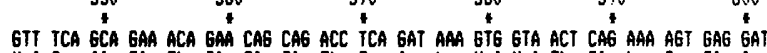

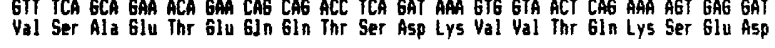

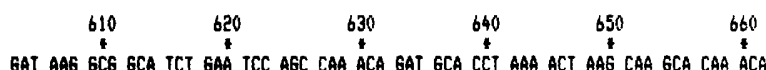
6AT AAG GCG GCA TCT GAA TCC AGC CAA ACA GAT GCA CCI AAA ACT AAG CAA GCA CAA ACA
ASP LYS Ala Ala Ser Glu Ser Ser GIn Thr ASP Ala Pro LYS Thr Lys GIn Ala Gli Thr

$\begin{array}{lllll}670 & 680 & 690 & 700 & 710\end{array}$ GAA CAA ACG CAG GCC CAA AGT CAG GCA AAC GTT GCT GAT ACA AGC ACT AGC ATA ACT A AAG Glu Gin Thr Gin Ala Gln Ser Gin Ala Asn Val Ala Asp Thr Ser Thr Ser lle Thr Lys $\begin{array}{llllll}730 & 740 & 750 & 760 & 770 & 780\end{array}$ GAA ACT CCT TCA CAA AAT ATT ACA ACA CAA GCC AAC ICT GAT GAC AAA ACA GTA ACA AAT $\begin{array}{llllll}790 & 800 & 810 & 820 & 830 & 840\end{array}$

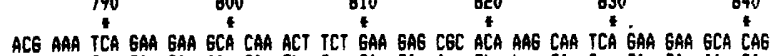
Thr Lys Ser Glu Glu Ala gin thr Ser Glu Glu Arg thr Lys Gin Ser Glu Glu Ala Gin $850 \quad 860 \quad 870 \quad 880 \quad 890 \quad 900$ ACT ACT GCT TCC AGT CÁ GCT ITA ACA CAag gCA AAA GCT GAA ITA ACA aAg CAA AGA CAA ACT ACT GCT SCC AGT GII Ala Leu Thr Gin Ala LYs Ala Glu Leu Thr LYS Gin Arg Gin

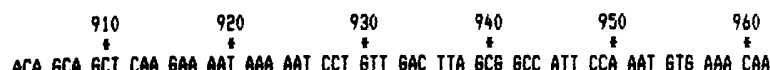

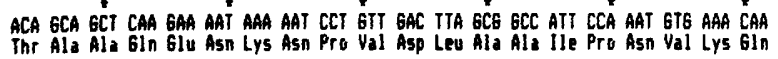
$\begin{array}{llllll}970 & 980 & 990 & 1000 & 1010 & 1020\end{array}$

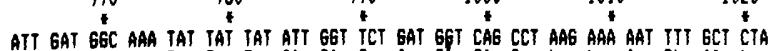

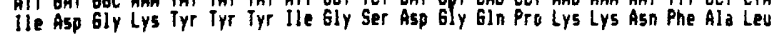
$1030 \quad$ Scal $\quad 1050 \quad 1060 \quad 1070 \quad 1080$ ACC GIT AAT AAC AAA GTA CTC TAC ITC GAT AAA AAT ACA GGT SCC ITA ACG GAC ACT TCT $1090 \quad 1100 \quad 1110 \quad 1120 \quad 1130 \quad 1140$ 1090 AhallI $1100 \quad$ Hpal $11100011120 \quad 1130 \quad 1140$

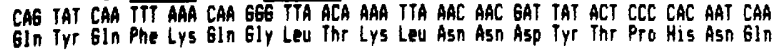
$\begin{array}{llllll}1150 & 1160 & 1170 & 1180 & 1190 & 1200\end{array}$

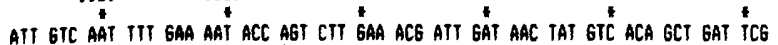

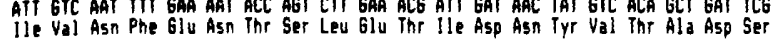

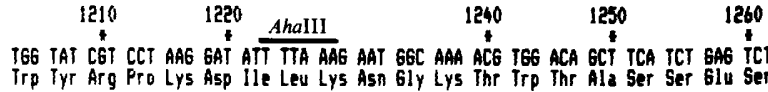
$\begin{array}{lllll}1270 & 1280 & 1300 & 1310 & 1320\end{array}$

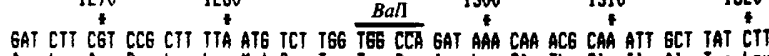
Asp Leu Arg Pro Leu Leu het Ser Irp Trp Pro Asp lys gin Thr Gin Ile Ale Tyr Le $\begin{array}{llllll}1330 & 1340 & -1350 & 1360 & 1370 & 1380\end{array}$ AAC TAC ATG AAC CAG CAA GGA CTT GGA ÁCT GGT GAA Aát TAC ACA GCA GAC AGC AGC CAA

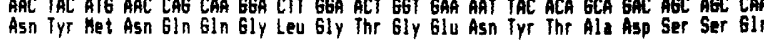
$\begin{array}{llllll}1390 & 1400 & 1410 & 1420 & 1430 & 1440\end{array}$ 6AA AGI CIC AAC CIT GCT GCA CAA ACC 6 II CAA GTT AAG ATT GAA ACT AAA ATT ICT CAA

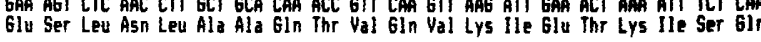

$\begin{array}{llllll}1450 & 1460 & 1470 & 1480 & 1490 & 1500\end{array}$ ACG CAG CÁ ACC CAG TGG CTG'CGT GAT AIT ATC AAT AGT ITI GIT AAA ACG CAA CCA AAT Thr Gin Gln Thr fln Trp leu ATg Asp Jle lle Asn Ser Phe yal Lys Thr filn Pro Asn

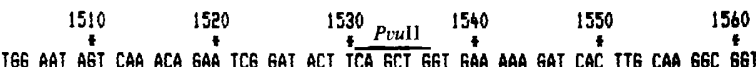

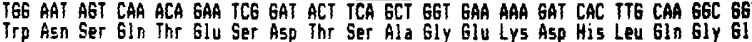
$\begin{array}{llllll}1570 \quad 1580 & 1590 & 1600 & 1610 & 1620\end{array}$ GCT CTG CTT TAT AGC AAC AGC GAT AAG ÁCA GCC TAT \&CT AAT ICC GAt TAC CGT CTT ITG Ala leu Leu Tyr Ser Asn Ser Asp lys thr Ala Tyr Ala Asn Ser Asp fyr Arg Leu Leu $\begin{array}{llllll}1630 & 1640 & 1650 & 1660 & 1670 & 1680\end{array}$ AAC CGC ACA CCA ACC AGT CAA ACG gGT ÄAA CCA AAA IAT IIT GAA GAC AAT TCT TCT

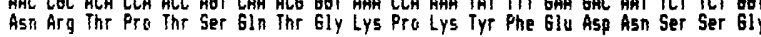
$\begin{array}{llllll}1690 & 1700 & 1710 & 1720 & 1730 & 1740\end{array}$ GGC IAT GAC ITC CTC CTA GCT AAT GAT AIT GAT AAT TCA AAT CCA GTG GTT CAA GCT GAA Gly Tyr Asp Phe Lev Leu Ala Asn Asp lile Asp Asn Ser Asn Pro Val Val Gin Ala Glu

$\begin{array}{llllll}1750 & 1760 & 1770 & 1780 & 1790 & 1800\end{array}$

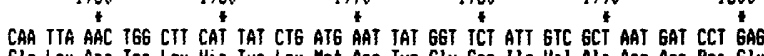
6In Leu Asn Itp leu His Tyr leu Het Asn Tyr Gly Ser lle Val Ala Asn Asp Pro Glu

$191018020 \quad 1830 \quad 1840 \quad 1850 \quad \frac{P}{*} \quad 1851$

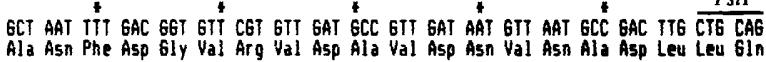
$\begin{array}{llllll}1870 & 1880 & 1890 & 1900 & 1990 & 1920\end{array}$ ATT GCT ICE GAC TAT TTG AAA GCC CAT IAT GGT GIT GAT AAG AGT GAG AAA AAT GCG ÁT lle ala Ser Asp Tyr Leu lys Ala His Tyr Gly Val Asp Lys Ser Glu Lys Asn Ala lle
$1930 \quad 1940$ HindIII ${ }^{1950} \quad 1960 \quad 1970 \quad 1980$

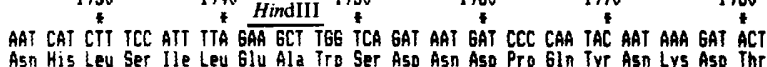
$\begin{array}{llllll}1990 & 2000 & 2010 & 2020 & 2030 & 2040\end{array}$ AAG GGI GCA CAA ITA CCG AIT GAT AAT AAA CTE CGC CTA TCG CIT ITA TAT GCT ITE ACG Lys Gly Ala Gin Leu PTo 11 Asp Asn lys Leu ATg Leu Ser Leu Leu Tyr Ala Leu Thr $\begin{array}{llllll}2050 & 2060 & 2070 & 2080 & 2090 & 2300\end{array}$

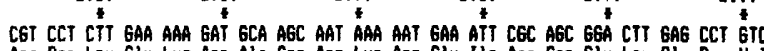
Arg Pro Leu Glu Lys Asp ala Ser Asn Lys Asn glu the Arg Ser Gly Leu Glu Pro Va ATA ACA AAT AGC ITG AAT AAC CGT TCA GCT GAA GGT AAA AAT AGT GAA CGT ATG GCT AAC Ile Thr Asn Ser Leu Asn Asn Arg Ser Ala Glu giy Lys Asn Ser Glu Arg het Ala Asn $2170 \quad 2180 \quad 2190 \quad 2200 \quad 2210 \quad 2220$ TAT AIT IIT ATC CGC GCT CAC GAC AGI GAA GTC CAA ACG GTT ATT GCT AAA ATI ATT AAC

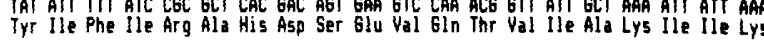
$2230 \quad 2240 \quad 2250 \quad 226022270$

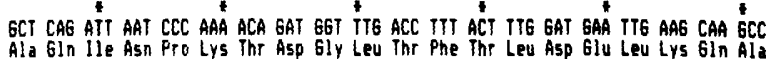
$\begin{array}{lllll}B g / I I & 2300 & 2310 & 2320 & 2330\end{array}$

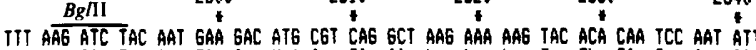

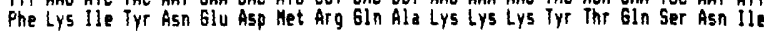
$2350 \quad 2360 \quad 2370 \quad 2380 \quad 2390 \quad 2400$

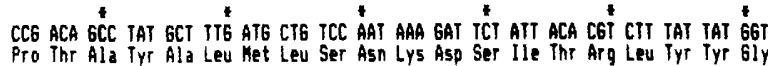

Fig. 2 (continued overleaf). Nucleotide sequence of the $4.9 \mathrm{~kb} \mathrm{XbaI-AhaIII} \mathrm{fragment} \mathrm{containing} \mathrm{the} \mathrm{intact} g f t D$ gene, and the deduced amino acid sequence of the GTF-S enzyme. The Shine-Dalgarno (SD) sequence is underlined (positions 423-427). The arrows indicate the positions of the three direct repeating units $\mathrm{A}, \mathrm{B}$, and $\mathrm{C}$. 


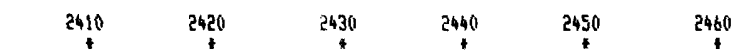

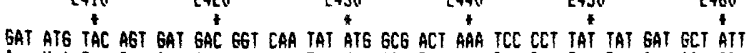
Asp het tyr Ser Asp Asp Gly Gin Tyr het Ala thr Lys Ser Pro Tyr Tyr Asp Ala lle

$$
2470 \quad 2480 \quad 2490 \quad 2500 \quad 2510 \quad 2520
$$

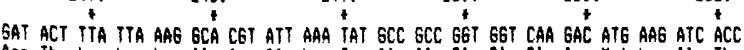
Asp thr Leu Leu LYS Ala Arg Ile LYS TYr Ala Ala Gly Gly Gin ASp het Lys lle Thr

$2530 \quad 2540 \quad V_{d e l} 2550 \quad 2560 \quad 2570 \quad 2580$

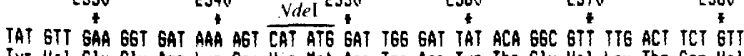
Tyr val Glu Gly Asp Lys Ser His het Asp Trp Asp Tyr ihr Gly Val Leu Thr Ser yal

$2590 \quad 2600 \quad 2610 \quad 2620 \quad 2630 \quad 2640$

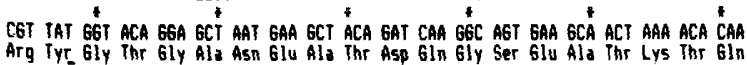
$\begin{array}{llllll}2650 & 2660 & 2670 & 2680 & 2690 & 2700\end{array}$

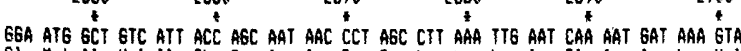
6ly het ala val Ile thr Ser Asn Asn Pro Ser Leu Lys Leu Asn Gln Asn Asp Lys yal

$2710 \quad 2720 \quad 2730 \quad 2740 \quad 2750 \quad 2760$

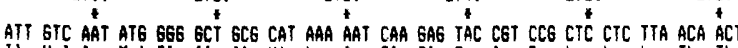

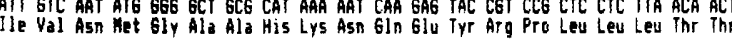

$2770 \quad 2780 \quad 2790 \quad 2800 \quad 2810 \quad 2820$

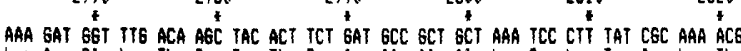
Lys Asp Gly Leu Thr Ser Tyr Thr Ser Asp Ala Ala Ala Lys Ser Leu Tyr Arg Lys Thr
$2830 \quad 2940 \quad 2850 \quad 2860 \quad 2870 \quad 2890$

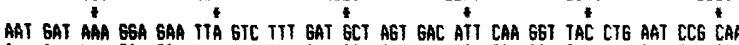

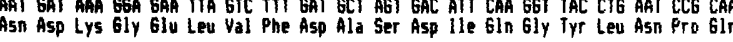

$2990 \quad 2900 \quad 2910 \quad 2920 \quad 2930 \quad 2940$

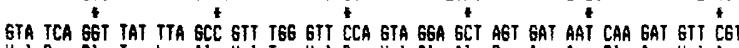
Val ser Gly Tyr Lew Ala val Trp val Pro val Giy Ala Ser Asp Asn GIn Asp val Arg

$\begin{array}{llllll}2950 & 2960 & 2970 & 2980 & 2990 & 3000\end{array}$ GTA GCA GCA AGC AAT AAG GCA AAT GCT ACT GGT CAA GTC TAC GAA TCA ICA AGT GCT CT Val Ala Ale Ser Asn Lys ala Asn Ala thr Gly Gln val Tyr Glu Ser Ser Ser Ala Leu

$\begin{array}{llllll}3010 & 3020 & 3030 & 3040 & 3050 & 3060\end{array}$

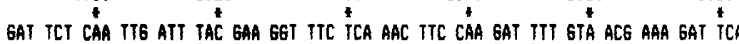
ASP Ser GIn Ley Ile Tyr Glu Gly Phe Ser ASn Phe Gin ASP Phe Yal thr LYS ASP Ser

$$
3070 \quad 3080 \quad 3090 \quad 3100 \quad 3110 \quad 3120
$$
GAC TAT ACT AAT AAG AAG AIT GCT CAA AAT GIC CAA CIC ITC AAA ICT TGG GGT GTC ACT Asp Tyr Thr Asn Lys Lys tle Ala Gin Asn yal gln Leu Phe Lys Ser Trp gly val thr

$\begin{array}{llllll}3130 & 3140 \quad 3150 \quad 3160 \quad \text { Xbal } 3180\end{array}$ TCC ITT GAA ATE GCA CCE CAA TAT GTC ICT TCT GAA GAT GGT ICT MTT XTA GAC TCT ATT

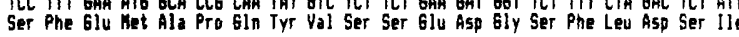

$3190 \quad 3200 \quad 3210 \quad 3220 \quad 3230 \quad 3240$

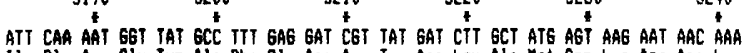
Ile gin Asn Gly fyr Ala Phe glu Asp Arg Tyr Asp LeU Ala het Ser Lys ASn ASn Lys

$3250 \quad 3260 \quad 3270 \quad 3280 \quad 3290 \quad 3300$ IAC GGT ICT CAG CAA GAC ATG ATT AAT GCA GTT AAA GCT CTE CAT AAÁ AGC GGT ATI CAG $3310 \quad 3330 \quad 3340 \quad 3350 \quad 3360$

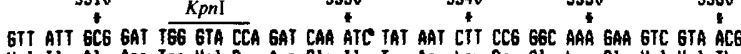
Val ile Ala ASP Irp Val Pro ASp Gin Ile Tyr ASn Lev Pro Gly Lys Glu Val Val Thr $\begin{array}{llllll}3370 & 3380 & 3390 & 3400 & 3410 & 3420\end{array}$ GCT ACA CGI GTC AAC GAT TAT GGT gAG İAT CGC AAA GAC TCT GAA AIC AAA AAT ACA CTC $\begin{array}{llllll}3430 & 3440 & 3450 & 3460 & 3470 & 3480\end{array}$ TAT GCT GCC AAC ACT A Ä AGT AAT GGT Iyr Ala Ala Asn Thr Lys Ser Asn Gly Lys Asp Tyr Gin Ala Lys Tyr Gly Gly Ala Phe

$3490 \quad 3500 \quad 3510 \quad 3520 \quad 3530 \quad 3540$ CIT AGT GAA CTC GCT GCT AAG TAC CCT ÁGT AIC TTT AAC CSC ACG CAA ATT TCA AAT GGT

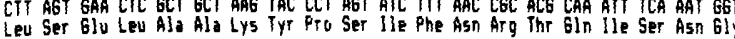

$3550 \quad 3560 \quad 3570 \quad 3580 \quad 3590 \quad 3600$

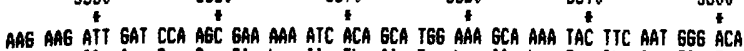
Lys Lys tle Asp Pro Ser Glu Lys Ile Thr Ala Irp Lys Ala Lys Tyr Phe Asn Gly Thr

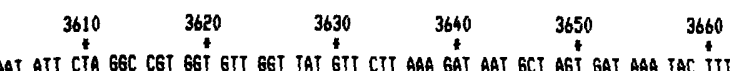

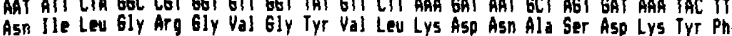

$3670 \quad 3680 \quad 3690 \quad 3700 \quad 3710$ HindIII 3720

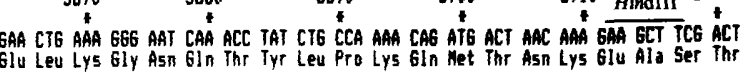
$\begin{array}{llllll}3730 & 3740 & 3750 & 3760 & 3770 & 3790\end{array}$

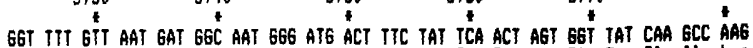

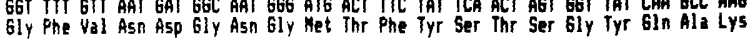

$3790 \quad 3800 \quad 3810 \quad 3820 \quad 3830 \quad$ Ball Nde1 AAC AGC ItI GTT CAA GAT GCC AAA GGA AAC TGG TAT TAC ITT GAT AAT AAT GGC CAT ATG Asn Ser phe Yal gln Asp Ala Lys gly Asn Trp Tyr Tyr phe Asp Asn Asn Giy His Met

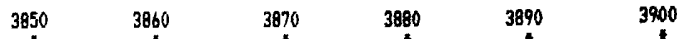

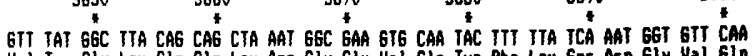
Val Tyr Gly Leu Glo Glo Leu Asn Gly Glu Val Gin Tyr phe Leu Ser ASA Giy val Gin

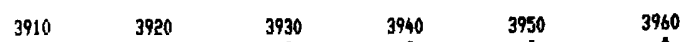
ITE CGT SAA TCT ITC TTE GAA AAC GCT GAT GGC AGC AAG AAC TAT TII GGT CAT CTA GGA Leu Arg glu Ser Phe Leu Glu Asn Ala Asp gly Ser Lys Ast Tyr Phe Gly His Leu Gly

$\begin{array}{llllll}3970 & 3980 & 3990 & 4000 & 4010 & 4020\end{array}$

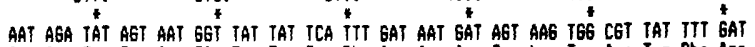
ASn Arg Tyr Ser Asn Gly Tyr Tyr Ser Phe ASp ASn Asp Ser Lys ITp Arg Tyr Phe ASP

$$
4030 \quad 4040 \quad 4050 \quad 4060 \quad 4070 \quad \text { ScaI } \quad B c \text { B I }
$$
GCC AGT GGA GTC ATG GCT GTA GGT ITT AAA ACA ATT AAC GGC AAT ACG CAS TAC TIT GAT Ala Ser Gly val het Ala val Giy Leu Lys Thr lle Asn Gly Asn Thr Gin Tyr Phe Asp $\begin{array}{llllll}4090 & 4100 & 4110 & 4120 & 4130 & 4140\end{array}$ CAA GAT GGI TAT CAA GIC AAA GGT GCT IGG ATA ACA GGC AGC GAT GGC AAA AAG CGT JAT Gin Asp Gly iyr Gin Val Lys Gly Ala ITp Ile Thr Gly Ser Asp Gly Lys lys Arg Tyr

$$
4150 \quad 4160 \quad 4170 \quad 4180 \quad \underset{4190 \mathrm{~A}}{4} 4200
$$

ITT GAT GAC EGA TCT GGA AAT ATE GCT G̈T AAT CGT İT GCA AAT GAT AAA AAC GGC EAI

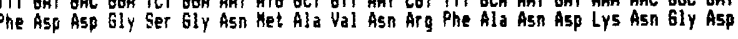

$4210 \quad 4220 \quad 4230 \quad 4240 \quad 4250 \quad 4260$ IEg TAC TAT TTC AAT TCA GAT GGC AIT irp Tys Tyr Leu Asn Ser Asp gly lle Ala Leu Val Gly Val Gin ihr lle Asn Gly Lys

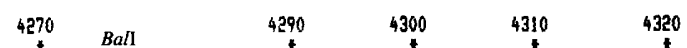

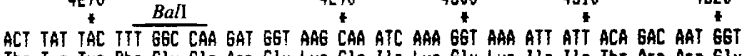
Thr Tyr Tyr Phe Gly gin Asp gly Lys Gin lie Lys gly Lys lle lle Thr Asp Asn Gly

$4330 \quad 4340 \quad 4350 \quad 4360 \quad 4370 \quad 4380^{\mathrm{B}}$ AAG CTG AAA TAT TIC CII GCC AAT TCA 'GGA GAA TTA GCA CGC AAT ATC ITT GCA ACA GAC Lys Leu Lys Tyr phe Leu Ala Asn Ser Gly Glu Leu Ala Arg Asn Ile Phe Ala Thr ASB

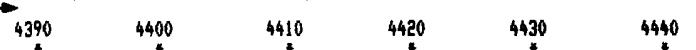
AGT CAA AAC AAT TGG TAT TAC III GGT ICA GAC GGI GIT GCE GIT ACA GSC AGT CAG ACA Ser Gin Asn Asn brp Tyr Tyr Phe Gly Ser Asp gly Val Ala Val Thr Gly Ser Gin Thr

$$
4450 \quad 4460 \quad 4470 \quad 4480 \quad 4490 \quad 4500
$$
ATT GCT GGT AAA AAG CTC IAT IIT GCA AGC GAC GGA AAA CAA GTC AAA GGC AGC IIT GTC $\begin{array}{lllll}4510 & 4520 & 4530 & 4540 & 4550\end{array}$

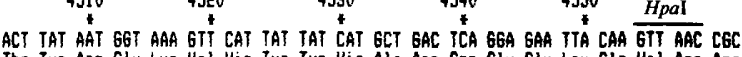
Thr Tyr Asn Gly LYs Yal His Tyr Tyr His Ala Asp Ser Gly glu Leu Gin Val Asn Arg
4570 C $4580 \quad 4590 \quad 4600 \quad 4610 \quad 4620$

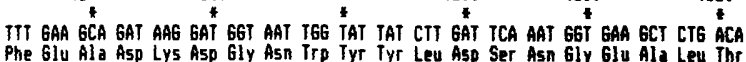

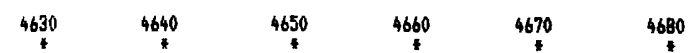
GGC AGC CAA CGC ATT AAC GAT CAG CGT GTC ITC ITT ACG CGA GAA GGA AAA CAA GIT AAA Giy Ser 6in Arg lle Asn Asp gin Arg Val Phe Phe ihr Arg Glu Gly Lys Gln Val Lys$$
\begin{array}{llllll}
4690 & 4700 & 4710 & 4720 & 4730 & 4740
\end{array}
$$

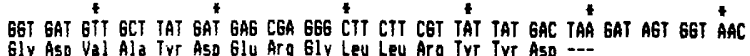
$4750 \quad 4760 \quad 4770 \quad 4780 \quad 4790 \quad 4800$

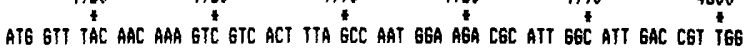
$4810 \quad 4820 \quad 4830 \quad 4840 \quad B C C 1 \quad 4950$

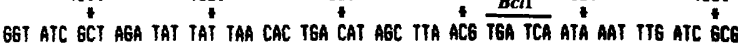

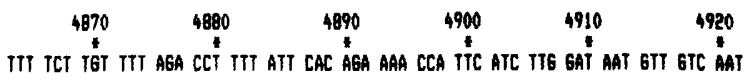
CII ITT TAA A 


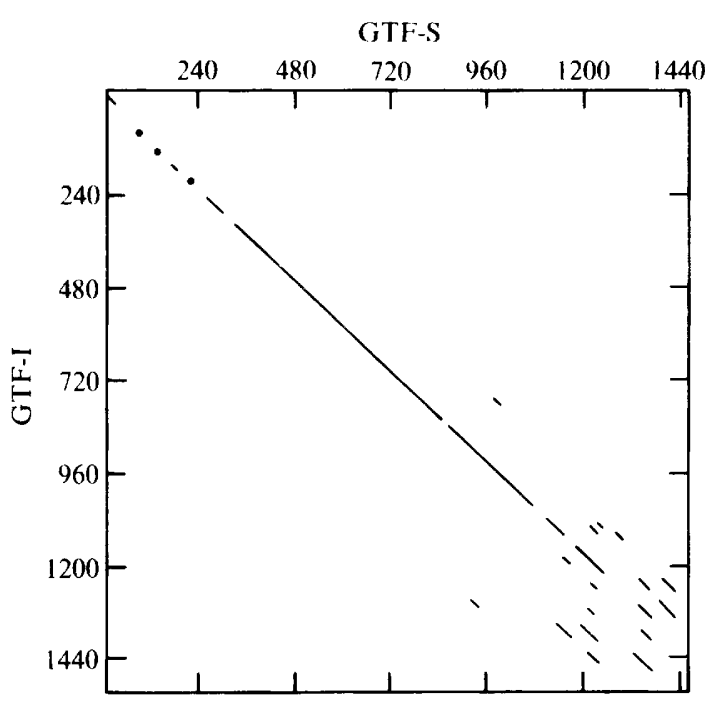

Fig. 3. Amino acid similarity matrix between the GTF-I and GTF-S proteins. Window sequences of six amino acids are compared and each point represents greater than $50 \%$ comparative similarity. Dots represent relatively short similar sequences.

both contain relatively long (65 and 49 amino acids respectively) highly homologous direct repeating units at the carboxyl termini (Shiroza et al., 1987; Ueda et al., 1988). Likewise, three repeating units consisting of sixtyfive amino acids were identified in the $\mathrm{COOH}$-terminal region of the GTF-S protein (corresponding to base positions 4128-4319, 4320-4511 and 4512-4700; Fig. 2). In addition, the direct repeating units of the GTF-I protein exhibited significant identity with the GTF-S sequence (Fig. 4). Furthermore, the GTF-SI protein repeating unit was less similar to that of the GTF-S protein than to the GTF-I repeat sequences (data not shown). All three enzymes also share significant but lower similarity with the GTF-I protein produced by $S$. downei (Ferretti et al., 1987).

\section{Signal sequence regions of $S$. mutans extracellular proteins}

The $g t f D$ gene is the fourth $S$. mutans extracellular protein gene to be sequenced in this laboratory. Therefore, it was of interest to compare the signal sequence regions of these proteins, in order to identify amino acids which might be important in secretion. A comparison of the amino acids in these regions from the three GTF proteins and from the extracellular fructosyltransferase (FTF) secreted by strain GS-5 (Shiroza \& Kuramitsu, 1988) revealed a number of similarities (Fig. 5). In addition to the initiator amino acid methionine, seven amino acid positions were identical within the first seventeen amino-terminal amino acids. This similarity between these regions of the GTF and FTF proteins from $S$. mutans is in marked contrast to the lack of identity displayed between the processed forms of the two groups of enzymes (T. Shiroza \& H. K. Kuramitsu, unpublished results). Comparison with the sequence of the GTF-I protein from another mutans streptococcus, S. downei strain $\mathrm{MFe} 28$ (Ferretti et al., 1987), indicated that six amino acid positions were conserved for all five proteins. All of these putative signal sequences contained an acidic amino acid at position 2, Arg at position 6, Lys at positions 8,11 and $13, \operatorname{Trp}$ at position 16, and Val at position 17. As might be expected for similar enzymes from the same strain, all three $S$. mutans GTF shared a number of other conserved amino acid positions (sixteen of the first thirty amino acids). In addition, the three GTF enzymes from $S$. mutans strains GS-5 together with the GTF-I protein from $S$. downei exhibited thirteen identical amino acid positions in this region.

\section{Discussion}

The deduced amino acid sequence of the GTF-S enzyme expressed by the $g t f D$ gene indicated that the protein is highly hydrophilic with an unprocessed molecular mass of approximately $159 \mathrm{kDa}$ (Fig. 2). This size is similar to that determined for both the GTF-I (Shiroza et al., 1987) and GTF-SI (Ueda et al., 1988) proteins. Purification of the GTF-S enzyme from either $S$. mutans or $E$. coli indicated two forms, with molecular masses of 140 and $155 \mathrm{kDa}$ (Hanada \& Kuramitsu, 1989). This would suggest that cleavage with the signal peptidase within the first $30-40$ amino acids is followed by proteolytic processing. However, the nature and location of these alterations remains to be determined for the GTF-S protein.

The predicted pI of 6.29 for the unprocessed GTF-S protein is somewhat more acidic than those for both the GTF-I and GTF-SI proteins (pI 6.87 and 9.4, respectively; Shiroza et al., 1987; Ueda et al., 1988). However, purification of the GTF-S enzyme from culture supernatant fluids of strain GS-5 produced a $140 \mathrm{kDa}$ protein with a pI of approximately 7.5 (Kuramitsu \& Wondrack, 1983). Therefore, it is likely that processing of the extracellular GTF-S protein in $S$. mutans is accompanied by modification of the primary translation product of the $g t f D$ gene to a more basic protein.

Since the three GTF enzymes secreted by $S$. mutans synthesize glucans differing primarily in the proportions of $\alpha-1,6-$ and $\alpha-1,3$-glucose linkages, it is not surprising that the proteins are similar to one another. The highest degree of similarity among the proteins exists within the central regions of the sequences (Fig. 3; Ueda et al., 1988). A comparison of amino acids corresponding to the $2.0 \mathrm{~kb}$ central DNA fragments (HindIII fragment of the 
(a) GTF-S

A GKKRYFDDGSGMMAVNRFANDKNGDWYYLNSDGIALVGVQTINGKTYYFGQDGKQIKGKIITDN

B GKLKYFLANSGELARNIFATDSQNNWYYFGSDGVAVTGSQTIAGKKLYFASDGKQVKGSFVTYN

C GKVHYYHADSGELQVNRFEADKDGNWYYLDSNGEALTGSQRINDQRVFFTREGKQVKGDVAYDE

(b) GTF-S and GTF-I

S-B GKLKYFLANSGELARNIFATDSQNNWYYFGSDGVAVTGSOTIAGKKLYFASDGKQVKGSFVTYN I-A GRISYYDGNSGDQIRNRFVRNAQGQWFYFDNNGYAVTGARTINGQLLYFRANGVQVKGEFVTDR
Fig. 4. Amino acid direct repeating units at the carboxyl termini of the GTF proteins. The amino acid direct repeating units for the $S$. mutans $g t f B$ and $g t f D$ gene products (GTF-I and GTF-S respectively) are depicted in single-letter code. Identical amino acid positions are depicted by stars. (a) The three direct repeating units of the GTF-S protein (see Fig. 2). (b) A comparison of the second GTF-S repeating unit (B) with the first repeating unit (A) of the GTF-I protein.

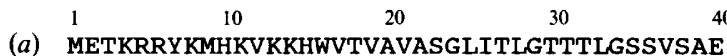
I I I I I
(b) MDKKVRYKLRKVKKRWVTVSVASAVMTLTTLSGGLVKADS
I I I I I
(c) MEKKVRFKLRKVKKRWVTVSIASAVVTLTSLSGSLVKADS
(d) METKVRKKMYKKGKFWVVATITTAMLTGIGLSSVQADEAN
(e) MEKNERFKMHKVKKRWVTISVASATMLASALGASVASADT

Fig. 5. Comparison of the signal sequence regions of mutans streptococcal extracellular proteins. Identical amino acid positions for all five proteins are indicated by connecting vertical lines. (a) $S$. mutans GTF-S; (b) S. mutans GTF-I; (c) S. mutans GTF-SI ; (d) S. mutans FTF; (e) $S$. downei GTF-I. The arrow depicts the signal cleavage site for the $S$. downei GTF-I as determined by amino acid sequencing (Ferretti et al., 1987).

gtfD gene) revealed approximately $95 \%$ sequence identity at the amino acid level between the GTF-I and GTFSI proteins. In this same region, $84 \%$ identity was determined between the GTF-I and GTF-S proteins. Such differences are consistent with the observations that both the GTF-I and GTF-SI enzymes synthesize primarily water-insoluble glucans (Aoki et al., 1986; Hanada \& Kuramitsu, 1989) while the GTF-S enzyme synthesizes water-soluble glucans (Hanada \& Kuramitsu, 1989). Comparison of the three $S$. mutans GTF sequences also reveal regions of sequence dissimilarity (Fig. 3).

Recent results have indicated that the carboxyl termini of both the GTF-I and GTF-SI proteins from $S$. mutans, as well as the GTF-I from $S$. downei, contain relatively long homologous direct repeating units (Shiroza et al., 1987; Ueda et al., 1988; Ferretti et al., 1987). The present results demonstrate that similar direct repeating units are present at the carboxyl terminus of the GTF-S protein. However, the similarity of the repeating units within the GTF-S protein (52\% identity between units $\mathrm{A}$ and $\mathrm{B}$; Fig. 4) was less than that observed between the homologous units in the GTF-I and GTF-SI proteins $(95 \%$ and $75 \%$ respectively; Shiroza et al., 1987; Ueda et al., 1988).

A comparison of the GTF-S signal sequence with three other $S$. mutans extracellular proteins revealed a number of identical amino acid positions (Fig. 5). Likewise, these four $S$. mutans signal sequence regions shared a number of amino acid positions with that of the GTF-I from $S$. downei. Comparison with the signal sequences of other streptococcal extracellular proteins, namely streptokinase (Malke et al., 1985) and group A streptococcus M protein (Hollingshead et al., 1986), did not reveal sequence similarity. Based on a limited comparison of the available sequences, the mutans streptococcal extracellular protein signal sequences appear to have conserved similar structural features. However, the role of individual amino acids in the secretion process remains to be elucidated.

Since the tandem $g t f B$ and $g t f C$ genes may be coordinately regulated (Ueda et al., 1988) and this genetic locus is not present near the gtfD gene (D. Perry \& H. K. Kuramitsu, unpublished results), it will be of interest to examine the regulation of expression of this latter gene.

This investigation was supported in part by Public Health Service grants DE-03258 and DE-06080 from the National Institutes of Health.

\section{References}

Aoki, H., Shiroza, T., Hayakawa, M., Sato, S. \& Kuramitsu, H. K. (1986). Cloning of a Streptococcus mutans glucosyltransferase gene coding for insoluble glucan synthesis. Infection and Immunity 53, 587594.

Ferretti, J. J., GilPin, M. K. \& Russell, R. R. B. (1987). Nucleotide sequence of a glucosyltransferase gene from Streptococcus sobrinus MFe28. Journal of Bacteriology 169, 4271-4278.

GibBons, R. J. \& van Houte, J. (1975). Dental caries. Annual Review of Medicine 26, 121-136.

Gilpin, M. L., Russell, R. R. B. \& Morrissey, P. (1985). Cloning and expression of two Streptococcus mutans glucosyltransferases in Escherichia coli K-12. Infection and Immunity 49, 414-416.

Hamada, S. \& Slade, H. D. (1980). Biology, immunology, and cariogenicity of Streptococcus mutans. Microbiological Reviews 44, $331-384$

HaNada, N. \& Kuramitsu, H. K. (1988). Isolation and characterization of the Streptococcus mutans gtfC gene, coding for the synthesis of both soluble and insoluble glucans. Infection and Immunity 56, 19992005. 
HANADA, N. \& KURAMiTSU, H. K. (1989). Isolation and characterization of the Streptococcus mutans gtfD gene, coding for primerdependent soluble glucan synthesis. Infection and Immunity 57, 2079 2085.

HaWley, D. K. \& McClure, W. R. (1983). Compilation and analysis of Escherichia coli promoter DNA sequences. Nucleic Acid Research 11, 2237-2255.

von HeIJNe, G. \& ABRaHMSON, L. (1989). Species specific variation in signal peptide design. Implications for protein secretion in foreign hosts. FEBS Letters 244, 439-441.

HENIKOFF, S. (1984). Unidirectional digestion with exonuclease III creates targeted breakpoints for DNA sequencing. Gene 28, 351-359.

Hollingshead, S. K., Fischetti, V. A. \& Scott, J. R. (1986) Complete nucleotide sequence of the type $6 \mathrm{M}$ protein of the group $\mathrm{A}$ streptococcus. Journal of Biological Chemistry 261, 1677-1686.

KURAMITSU, H. K. \& WONDRACK, L. (1983). Insoluble glucan synthesis by Streptococcus mutans serotype c strains. Infection and Immunity $\mathbf{4 2}$, 763-770.

LOESCHE, W. J. (1986). Role of Streptococcus mutans in human dental decay. Microbial Reviews 50, 353-380.

Malke, H., RoE, B. \& Ferretti, J. J. (1985). Nucleotide sequence of the streptokinase gene from Streptococcus equisimilis H46A. Gene 34, 357-362.
Russell, R. R. B., Shiroza, T., Kuramitsu, H. K. \& Ferretti, J. J. (1988). Homology of glucosyltransferase gene and protein sequences from Streptococcus sobrinus and Streptococcus mutans. Journal of Dental Research 67, 543-547.

SANGer, F., Nicklen, S. \& Coulson, A. R. (1977). DNA sequencing with chain-terminating inhibitors. Proceedings of the National Academy of Sciences of the United States of America 74, 5463-5467.

Shimamura, A., Tsumori, H. \& MUKaSa, H. (1983). Three kinds of extracellular glucosyltransferases from Streptococcus mutans 6715 (serotype g). FEBS Letters 157, 79-81.

Shine, J. \& Dalgarno, L. (1974). The $3^{\prime}$ terminal sequence of Escherichia coli $16 \mathrm{~S}$ ribosomal RNA: complementarity to nonsense triplets and ribosome binding sites. Proceedings of the National Academy of Sciences of the United States of America 71, 1342-1346.

ShIROZA, T. \& KURAMITSU, H. K. (1988). Sequence analysis of the Streptococcus mutans fructosyltransferase gene and flanking regions. Journal of Bacteriology 170, 810-816.

ShIroza, T., Ueda, S. \& Kuramitsu, H. K. (1987). Sequence analysis of the gtfB gene from Streptococcus mutans. Journal of Bacteriology $169,4263-4270$.

Ueda, S., Shiroza, T. \& KURAmitsu, H. K. (1988). Sequence analysis of the gtfC gene from Streptococcus mutans GS-5. Gene 69, 101-109. 